\title{
Mid-range adiabatic wireless energy transfer via a mediator coil
}

\author{
Andon A. Rangelov ${ }^{1,2}$ and Nikolay V. Vitanov ${ }^{1}$ \\ ${ }^{1}$ Department of Physics, Sofia University, 5 James Bourchier blvd, 1164 Sofia, Bulgaria \\ ${ }^{2}$ Corresponding author: rangelov@phys.uni-sofia.bg
}

(Dated: April 19, 2022)

\begin{abstract}
A technique for efficient mid-range wireless energy transfer between two coils via a mediator coil is proposed. By varying the coil frequencies three resonances are created: emitter-mediator (EM), mediator-receiver (MR) and emitter-receiver (ER). If the frequency sweeps are adiabatic and such that the ER resonance precedes the MR resonance, the energy flows sequentially along the chain emitter-mediator-receiver. If the MR resonance precedes the ER resonance, then the energy flows directly from the emitter to the receiver via the ER resonance; then the losses from the mediator are suppressed. This technique is robust to noise, resonant constraints and external interferences.
\end{abstract}

PACS numbers:

Since the pioneering work of Tesla the search for wireless power transfer is a hot topic for its vast potential to substitute electrical cables and batteries. Until very recently traditional magnetic induction, wherein two conductive coils induct each other, has been used to transfer energy wirelessly over very short distances only. The coils do not make direct electrical contact with each other but they must be very close because the efficiency of power transfer drops by orders of magnitude when the distance between the coils becomes larger than their sizes.

Recently, some novel ideas for medium-range wireless energy transfer have emerged $[1,2]$. Specially designed magnetic resonators achieve strong coupling between the coils that enable efficient energy transfer over distances much larger than the size of the coils $[1,2]$. This technique, which demands the same frequencies of the emitter and receiver coils, can be made more robust to variations of the coil parameters by sweeping the emitter frequency through resonance with the receiver frequency (or vice versa) [3] in a fashion reminiscent of adiabatic passage through a level crossing in quantum physics [4].

A recent theoretical paper [5] proposed a setup of two identical coils (emitter and receiver) strongly coupled to an intermediate coil (mediator) of different properties but with the same intrinsic frequency. The energy transfer occurs due to adiabatic following of an instantaneous ("dark") eigenstate of the three-coil system, an idea similar to the quantum three-state technique of stimulated Raman adiabatic passage (STIRAP) [6-8]. In this technique both the emitter and the receiver should be rotated in synchro in order to engineer time-dependent couplings that reduce the energy stored at the mediator [5]. It is, however, crucial to maintain an exact resonance between the emitter and receiver frequencies [5]; a frequency mismatch, e.g., due to differences the coils or random noise (which may be caused by external objects near the coils), reduces the transfer efficiency significantly.

In this paper, we propose a different approach to wireless energy transfer between two coils via a mediator coil by using the ideas of adiabatic population transfer in a three-state quantum system with crossing energies [9-11]. This technique promises to be both efficient and robust

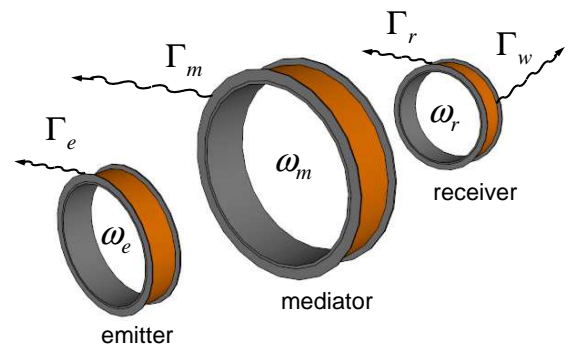

FIG. 1: (Color online) Three coils with intrinsic frequencies $\omega_{\mathrm{e}}, \omega_{\mathrm{m}}$ and $\omega_{\mathrm{r}}$. The large size of the mediator leads to strong couplings with the others coils, but also to larger loss rate $\Gamma_{\mathrm{m}}$ than the loss rates $\Gamma_{\mathrm{e}}$ and $\Gamma_{\mathrm{r}}$ of the emitter and receiver.

against variations of the parameters, such as the intrinsic frequencies of the coils and the couplings between them.

We consider three parallel helix coils as shown in Fig. 1: two small coils - an emitter and a receiver - and a large mediator, which induces strong couplings. We follow the description of the coupled mode theory as presented by Hamam et al. [5]. The energy transfer, in the strongcoupling regime, is described by a set of three coupled differential equations (in matrix form) [5],

$$
i \frac{d \mathbf{A}(t)}{d t}=\mathbf{H}(t) \mathbf{A}(t)
$$

where $\mathbf{A}=\left[a_{\mathrm{e}}(t), a_{\mathrm{m}}(t), a_{\mathrm{r}}(t)\right]^{T}$ and

$$
\mathbf{H}=\left[\begin{array}{ccc}
\omega_{\mathrm{e}}-i \Gamma_{\mathrm{e}} & \kappa_{\mathrm{em}} & 0 \\
\kappa_{\mathrm{em}} & \omega_{\mathrm{m}}-i \Gamma_{\mathrm{m}} & \kappa_{\mathrm{mr}} \\
0 & \kappa_{\mathrm{mr}} & \omega_{\mathrm{r}}-i \Gamma_{\mathrm{r}}-i \Gamma_{\mathrm{w}}
\end{array}\right] .
$$

Here $a_{\mathrm{e}}(t), a_{\mathrm{m}}(t)$ and $a_{\mathrm{r}}(t)$ are defined so that the energies contained in the emitter, the mediator and the receiver are, respectively, $\left|a_{\mathrm{e}}(t)\right|^{2},\left|a_{\mathrm{m}}(t)\right|^{2}$ and $\left|a_{\mathrm{r}}(t)\right|^{2}$, while $\Gamma_{\mathrm{e}}, \Gamma_{\mathrm{m}}$ and $\Gamma_{\mathrm{r}}$ are the corresponding intrinsic loss rates (due to absorption and radiation), and $\omega_{\mathrm{e}}(t), \omega_{\mathrm{m}}(t)$ and $\omega_{\mathrm{r}}(t)$ are the corresponding intrinsic frequencies. The extraction of work from the receiver is described by the term $\Gamma_{\mathrm{w}}$. The coupling coefficient between the emitter and the mediator is $\kappa_{\mathrm{em}}$ and the one between the 
mediator and the receiver is $\kappa_{\mathrm{mr}}$. The null elements in the matrix $\mathbf{H}(t)$ derive from the assumption that the mediator is much larger than the emitter and the receiver; hence the direct emitter-receiver coupling is negligible compared to the couplings $\kappa_{\mathrm{em}}$ and $\kappa_{\mathrm{mr}}$ and is neglected.

The efficiency of the energy transfer is described by the efficiency coefficient $\eta$ [1-3], which is the ratio between the work extracted from the receiver for the time interval $t_{i}-t$ divided by the total energy (absorbed and radiated) for the same time interval,

$$
\eta=\frac{\Gamma_{\mathrm{w}} \mathcal{E}_{\mathrm{r}}(t)}{\Gamma_{\mathrm{e}} \mathcal{E}_{\mathrm{e}}(t)+\Gamma_{\mathrm{m}} \mathcal{E}_{\mathrm{m}}(t)+\left(\Gamma_{\mathrm{r}}+\Gamma_{\mathrm{w}}\right) \mathcal{E}_{\mathrm{r}}(t)},
$$

where $\mathcal{E}_{k}(t)=\int_{t_{i}}^{t}\left|a_{k}(t)\right|^{2} \mathrm{~d} t(k=\mathrm{e}, \mathrm{m}, \mathrm{r})$.

Equation (1) is identical to the time-dependent Schrödinger equation for a three-state quantum system which is studied in great detail $[7,8]$; the vector $\mathbf{A}(t)$ and the driving matrix $\mathbf{H}(t)$ correspond to the quantum state vector and the Hamiltonian, respectively. By definition, in the adiabatic limit the system stays in an eigenvector of $\mathbf{H}(t)$. We first assume that the loss rates $\Gamma_{\mathrm{e}}, \Gamma_{\mathrm{m}}, \Gamma_{\mathrm{r}}$ and $\Gamma_{\mathrm{w}}$ are all zero; then the quantity $|\mathbf{A}(t)|^{2}=\left|a_{\mathrm{e}}(t)\right|^{2}+\left|a_{\mathrm{m}}(t)\right|^{2}+\left|a_{\mathrm{r}}(t)\right|^{2}$ is conserved, like the total population in a coherently driven quantum system.

The key of our proposal is the assumption that the frequency of the mediator coil is fixed, while the frequencies of the emitter and receiver coils change in time in opposite directions,

$$
\begin{aligned}
\omega_{\mathrm{e}}(t) & =\omega_{\mathrm{m}}+\delta+\alpha^{2} t, \\
\omega_{\mathrm{m}} & =\text { const }, \\
\omega_{\mathrm{r}}(t) & =\omega_{\mathrm{m}}+\delta-\alpha^{2} t,
\end{aligned}
$$

where $\delta$ is a suitably chosen static frequency offset, which controls the energy flow. For the sake of generality, we take hereafter $\alpha>0$ as the unit of frequency and $1 / \alpha$ as the unit of time. We assume that the fixed mediator frequency $\omega_{\mathrm{m}}$ is much larger than the terms $\delta \pm \alpha^{2} t$ in $\omega_{\mathrm{e}}(t)$ and $\omega_{\mathrm{r}}(t)$; therefore the couplings $\kappa_{\mathrm{em}}$ and $\kappa_{\mathrm{mr}}$ can be assumed constant for the sake of simplicity. Taking the variations of the couplings due to the frequency sweeps into account does not change the results significantly.

For $\delta \neq 0$, the intrinsic frequencies of the three coils cross each other at three different instants of times, thereby creating a triangle crossing pattern [9-11], shown in Fig. 2 (top frames). For $\delta=0$, the three frequencies cross at the same instant of time, thereby creating a bowtie crossing (frame B) [12]. These crossing patterns allow us to design recipes for efficient adiabatic wireless energy transfer, in analogy to adiabatic passage techniques in quantum physics [7-11].

The proposed technique is illustrated in Fig. 2. The top frames show the evolution of the intrinsic frequencies of the three coils $\omega_{k}(t)(k=\mathrm{e}, \mathrm{m}, \mathrm{r})$ and the eigenvalues $\varepsilon_{n}(t)(n=1,2,3)$ (the eigenfrequencies) of $\mathbf{H}(t)$ of Eq. (1). The three columns of frames differ by the offset frequency $\delta: \delta>0$ in the left column, $\delta=0$ in the middle
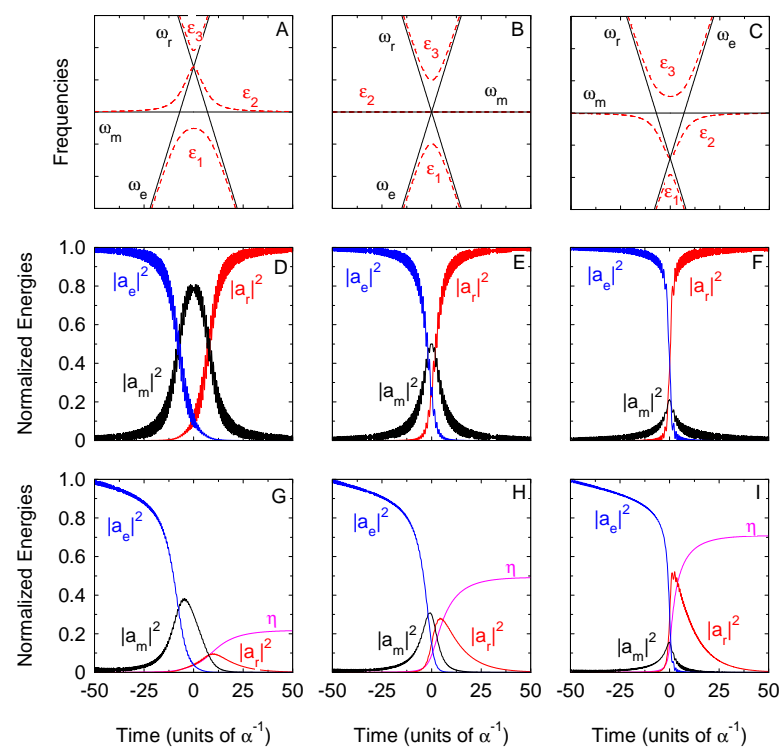

FIG. 2: (Color online) Wireless energy transfer. Left column: sequential transfer $(\delta=7 \alpha)$; middle column: bow-tie transfer $(\delta=0)$; right column: direct transfer $(\delta=-7 \alpha)$. Top frames: Diagonal elements (solid lines) and eigenvalues (dashed lines) of $\mathbf{H}$ of Eq. (1). Middle frames: The energies in the coils calculated numerically from Eq. (1) with no losses, $\Gamma_{\mathrm{e}}=\Gamma_{\mathrm{m}}=$ $\Gamma_{\mathrm{r}}=\Gamma_{\mathrm{w}}=0$ for $\kappa_{\mathrm{em}}=\kappa_{\mathrm{mr}}=3.5 \alpha$. Bottom frames: The energies in the coils and the efficiency coefficient $\eta$ for $\Gamma_{\mathrm{e}}=$ $\Gamma_{\mathrm{r}}=0.001 \alpha, \Gamma_{\mathrm{m}}=\Gamma_{\mathrm{w}}=0.05 \alpha$.

column and $\delta<0$ in the right column. In the beginning and the end each eigenfrequency $\varepsilon_{n}(t)$ coincides with one of the intrinsic frequencies $\omega_{k}(t)$ of the coils, while in between each $\varepsilon_{n}(t)$ is a superposition of $\omega_{\mathrm{e}}(t), \omega_{\mathrm{m}}$ and $\omega_{\mathrm{r}}(t)$. In the adiabatic limit, the system follows an eigenstate of $\mathbf{H}(t)$ and the eigenfrequency of the system at any instant of time is equal to the (time-dependent) eigenfrequency in which it is initially, i.e. $\varepsilon_{1}(t)$. However, the composition of $\varepsilon_{1}(t)$ is different for different $\delta$ because the topology of the crossings differ.

For $\delta>0$ (left column of frames), the frequency resonances occur in the intuitive manner: first the emittermediator resonance and then the mediator-receiver resonance. The eigenfrequency $\varepsilon_{1}(t)$ is initially nearly equal to $\omega_{\mathrm{e}}$, at intermediate times to $\omega_{\mathrm{m}}$ and in the end to $\omega_{\mathrm{r}}$. Consequently, the energy flows sequentially from the emitter to the mediator and then to the receiver coils; this sequential flow is demonstrated in frame $\mathrm{D}$. This two-step scheme extends the single-step adiabatic wireless energy transfer proposed earlier [3].

For $\delta=0$ (middle column of frames) - the bow-tie crossing - all resonances occur at the same time. The composition of $\varepsilon_{1}(t)$ is similar as for $\delta>0$, the difference being the lesser contribution of the mediator frequency $\omega_{\mathrm{m}}$ at intermediate times. Consequently, the mediator receives less transient energy, as seen in frame $\mathrm{E}$.

For $\delta<0$ (right column of frames), the frequency resonances occur in a counterintuitive manner, with the 
mediator-receiver resonance occurring before the emittermediator resonance. In fact, the system never comes close to the "nearest-neighbor" emitter-mediator and mediator-receiver resonances but it passes through the direct emitter-receiver resonance (frame $\mathrm{C}$ ). The eigenfrequency $\varepsilon_{1}(t)$ remains very far from the mediator frequency $\omega_{\mathrm{m}}$; consequently, very little energy attends transiently the mediator coil, as seen in frame F. This feature is reminiscent of the energy transfer in the STIRAPbased technique [5].

The coil parameters in Fig. 2 - the intrinsic frequencies, the couplings and the sweep rate - are chosen in a such way that in the absence of losses the evolution is adiabatic and hence the energy is transferred almost completely to the receiver coil in the end, as seen in frames $\mathrm{D}, \mathrm{E}$ and $\mathrm{F}$ of Fig. 2. However, the temporary transfer of energy to the mediator coil makes the energy transfer efficiency very different in the presence of losses, as seen in the bottom frames of Fig. 2. For $\delta>0$, a large amount of energy visits the mediator where is subjected to strong dissipation and most of it is lost before it has the chance to reach the receiver (frame $\mathrm{G}$ ). For $\delta=0$, less energy attends the mediator coil and hence some more energy reaches the receiver (frame $\mathrm{H}$ ). For $\delta<0$, only little energy visits the mediator coil and a significant amount of energy is transferred to the receiver (frame I).

We therefore identify the direct adiabatic path, occurring for $\delta<0$, as the optimal path for energy transfer from the emitter to the receiver.

Next we turn our attention to the conditions for adiabatic evolution in the three distinct cases $\delta>0, \delta=0$ and $\delta<0$. By using the formula for the transition probability for the Landau-Zener-Stückelberg-Majorana (LZSM) model [4], $p=1-\exp \left(-2 \pi \kappa^{2} / \alpha^{2}\right)$, we find that the condition for transition probability larger than $1-\epsilon$ at each crossing reads

$$
\frac{\kappa}{\alpha}>\sqrt{\frac{\ln (1 / \epsilon)}{2 \pi}}
$$

where $\kappa=\kappa_{\mathrm{em}}$ for the emitter-mediator resonance and $\kappa=\kappa_{\mathrm{mr}}$ for the mediator-receiver resonance. For the bow-tie crossing, the condition is (for $\kappa_{\mathrm{em}}=\kappa_{\mathrm{mr}}$ ) [12]

$$
\frac{\kappa}{\alpha}>\sqrt{\frac{\ln (1 / \epsilon)}{\pi}} .
$$

For the direct emitter-receiver resonance, we can use the LZSM formula again, by taking into account that the sweep rate is $2 \alpha$ and the effective emitter-receiver coupling is $\kappa=\sqrt{\kappa_{\mathrm{em}}^{2}+\kappa_{\mathrm{mr}}^{2}+\delta^{2} / 4}-\delta / 2[11]$,

$$
\frac{\kappa}{\alpha}>\sqrt{\frac{2 \ln (1 / \epsilon)}{\pi}}
$$

In addition to these conditions, efficient energy transfer requires that the loss rates be small compared to the couplings and the interaction time $T$,

$$
\Gamma_{k} \ll 1 / T, \kappa .
$$

One can readily verify that all these conditions are satisfied for the parameters in Fig. 2.

In conclusion, the proposed technique for adiabatic wireless energy transfer between an emitter and a receiver coil via a larger mediator coil allows one to transfer energy with high efficiency by varying the intrinsic frequencies of the coils in the counterintuitive way, in which the mediator-receiver resonance occurs before the emittermediator resonance. Then only a small amount of energy visits the mediator and hence the losses from the mediator are minimized. The presence of a large mediator coil allows one to increase the coil-coil couplings and therefore to increase the distance between the emitter and the receiver compared to the simple two-coil emitter-receiver setup [3]. Compared to the STIRAP-based energy transfer technique [5], the present technique does not require identical coil frequencies and time-varying couplings, and therefore it may be easier to implement.

This work is supported by the European Commission project FASTQUAST, and the Bulgarian National Science Fund grants D002-90/08 and DMU-03/103.
[1] A. Kurs, A. Karalis, R. Moffatt, J. D. Joannopoulos, P. Fisher, and M. Soljačić, Science 317, 83 (2007).

[2] A. Karalis, J. D. Joannopoulos, and M. Soljačić, Ann. Phys. 323, 34 (2008).

[3] A. A. Rangelov, H. Suchowski, Y. Silberberg, and N. V. Vitanov, Ann. Phys. 326, 626 (2011).

[4] L. D. Landau, Physik Z. Sowjetunion 2, 46 (1932); C. Zener, Proc. R. Soc. Lond. Ser. A 137, 696 (1932); E. C. G. Stückelberg, Helv. Phys. Acta 5, 369 (1932); E. Majorana, Nuovo Cimento 9, 43 (1932).

[5] R. E. Hamam, A. Karalis, J. D. Joannopoulos, and M. Soljačić, Ann. Phys. 324, 1783 (2009).

[6] U. Gaubatz, P. Rudecki, S. Schiemann, and K. Bergmann, J. Chem. Phys. 92, 5363 (1990).

[7] N. V. Vitanov, T. Halfmann, B. W. Shore, and K.
Bergmann, Annu. Rev. Phys. Chem. 52, 763 (2001).

[8] N. V. Vitanov, M. Fleischhauer, B. W. Shore, and K. Bergmann, Adv. At. Mol. Opt. Phys. 46, 55 (2001).

[9] B. Broers, L. D. Noordam, and H. B. van Linden van den Heuvell, Phys. Rev. A 46, 2749 (1992).

[10] R. G. Unanyan, N.V. Vitanov, and K. Bergmann, Phys. Rev. Lett. 87, 137902 (2001).

[11] S. S. Ivanov and N. V. Vitanov, Phys. Rev. A 77, 023406 (2008).

[12] C. E. Carroll and F. T. Hioe, J. Phys. A: Math. Gen. 19, 1151 (1986); C. E. Carroll and F. T. Hioe, J. Phys. A: Math. Gen. 19, 2061 (1986); V. N. Ostrovsky and H. Nakamura, J. Phys. A 30, 6939 (1997). 